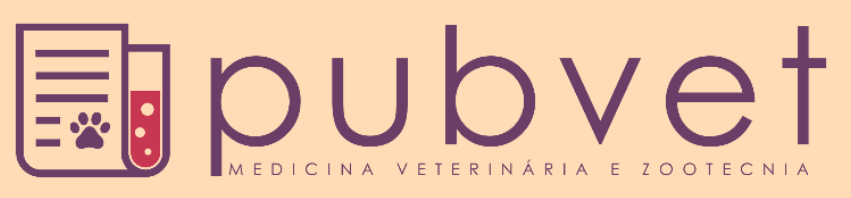

https://doi.org/10.31533/pubvet.v15n010a927.1-6

\title{
Perfil dos tutores e a importância da correta nutrição dos animais de companhia no estado de Rondônia
}

\author{
Denise Rufino Bragança ${ }^{1 *} \bullet$, Edicarlos Oliveira Queiroz $^{2} \bullet$, Raul Dirceu Pazdiora ${ }^{2} \oplus$ (D), Bruna \\ Rafaela Caetano Nunes Pazdiora ${ }^{20}$
}

${ }^{1}$ Bacharela em Ciências Agrárias - Produção Animal. Universidade Federal de Rondônia. Presidente Médici, Rondônia, Brasil. ${ }^{2}$ Professor adjunto da Universidade Federal de Rondônia, Presidente Médici, Rondônia, Brasil.

*Autor para correspondência, bragancad3@gmail.com

\begin{abstract}
Resumo. A população de pequenos animais chegou a 52,2 milhões de caninos e 22,1 milhões de felinos, os cães e gatos coabitam com os seres humanos a milhares de anos e as pessoas que convivem de forma saudável com seus pets tem um menor índice de depressão, essa ligação humano/animal vem trazendo benefícios a humanidade e com isso os cães e os gatos estão sendo considerados pelos tutores parte da família. A alimentação natural vem conquistando o mundo dos pets principalmente por não conter produtos químicos, conservantes artificiais e a matéria prima ser de fácil aquisição. Os dados foram adquirindo por meio de plataforma virtual e se constituiu a partir de questionário que se dispôs de três questões sobre o perfil do tutor: sexo, faixa etária e renda por salário-mínimo, duas questões para saber a população de cães/gatos e três últimas questões: se o tutor já consultou algum profissional em nutrição pet, se o mesmo gostaria de levar o seu animal ao nutricionista para obter uma dieta balanceada e quantos ele estaria disposto a pagar por uma consulta. Foram obtidas 109 respostas $68,8 \%$ de mulheres, $28,4 \%$ de homens e $2,8 \%$ tutor não binário. A maior parte das pessoas que participaram eram jovens com idade entre 18 e 33 anos. Das pessoas entrevistadas apenas 8 indivíduos não tinham cães e já para os gatos observa-se que 63 pessoas não possuem essa espécie em seu domicílio. a maioria dos tutores nunca consultou um profissional em nutrição pet para obter informações do que melhor se adequa a dieta deles. Foi observado a importância do perfil do tutor em relação a população de cães e gatos, ainda a conscientização sobre a necessidade de consultar um profissional antes de adquirir qualquer dieta nova no mercado para manter a saúde e o bemestar dos animais de companhia.
\end{abstract}

Palavras-chave: Manejo nutricional, entrevista, pets, consulta profissional

\section{Profile of tutors and the importance of correct nutrition for companion animals in the Rondonia state}

Abstract. The population of small animals reached 52.2 million canines and 22.1 million felines, dogs and cats have cohabited with humans for thousands of years and people who live in a healthy way with their pets have a lower rate of depression, this human/animal connection has brought benefits to humanity and with that the dogs and cats are being considered by the tutors as part of the family. Natural food has been conquering the world of pets mainly because it does not contain chemicals, artificial preservatives and the raw material is easy to acquire. The data were acquired through a virtual platform and consisted of a questionnaire that had 3 questions about the tutor's profile: sex, age group and minimum wage income, 2 questions to know the population of dogs/cats and 3 last questions: if the tutor has already consulted a professional in pet nutrition, if he would like to take his pet to the nutritionist to obtain a balanced diet and how many he would be willing 
to pay for an appointment. 109 responses were obtained, $68.8 \%$ women, $28.4 \%$ men and $2.8 \%$ non-binary tutor. Most of the people who participated were young people aged between 18 and 33 years old. of the people interviewed, only 8 individuals did not have dogs and for cats, it is observed that 63 people do not have this species at home. most tutors have never consulted a pet nutrition professional for information on what best suits their diet. The importance of the tutor's profile in relation to the population of dogs and cats was observed, as well as the awareness of the need to consult a professional before purchasing any new diet on the market to maintain the health and well-being of pets.

Keywords: Nutritional management, interview, pets, professional consultation

\section{Perfil de los tutores y la importancia de una correcta nutrición de las mascotas en el estado de Rondônia}

Resumen. La población de animales pequeños alcanzó los 52,2 millones de caninos y 22,1 millones de felinos, los perros y gatos han convivido con los humanos durante miles de años y las personas que conviven de forma saludable con sus mascotas tienen una menor tasa de depresión, esta conexión humano/animal ha traído beneficios a la humanidad y con eso los perros y gatos están siendo considerados por los tutores como parte de la familia. La comida natural ha ido conquistando el mundo de las mascotas principalmente porque no contiene químicos, conservantes artificiales y la materia prima es fácil de adquirir. Los datos se adquirieron a través de una plataforma virtual y consistieron en un cuestionario que contó con 3 preguntas sobre el perfil del tutor: sexo, grupo de edad y salario mínimo, 2 preguntas para conocer la población de perros / gatos y 3 últimas preguntas: si el tutor ya consultó a un profesional en nutrición de mascotas, si le gustaría llevar a su mascota al nutricionista para obtener una dieta balanceada y cuántas estaría dispuesto a pagar por una consulta. Se obtuvieron 109 respuestas, $68,8 \%$ mujeres, $28,4 \%$ hombres y $2,8 \%$ tutor no binario. La mayoría de las personas que participaron fueron jóvenes entre 18 y 33 años. De las personas entrevistadas, solo 8 individuos no tenían perros y para gatos, se observa que 63 personas no tienen esta especie en casa. la mayoría de los tutores nunca han consultado a un profesional en nutrición de mascotas para obtener información sobre lo que mejor se adapta a su dieta. Se observó la importancia del perfil del tutor con relación a la población de perros y gatos, así como la conciencia de la necesidad de consultar a un profesional antes de adquirir cualquier nueva dieta en el mercado para mantener la salud y el bienestar de las mascotas.

Palabras clave: Manejo nutricional, entrevista, mascotas, consulta profesional

\section{Introdução}

O Brasil tem umas das maiores populações mundiais de cães e gatos (ABINPET, 2021). Essa população de pequenos animais chegou a 52,2 milhões de caninos e 22,1 milhões de felinos (IBGE, 2020). Os animais de estimação convivem com os seres humanos a milhares de anos e proporciona aos tutores muitos benefícios psicológico, fisiológicos e social (Lima \& Luna, 2012), estudos mostram que pessoas que convivem de forma saudável com os animais tem menor índice de depressão e estresse, ainda melhora a qualidade de vida de pessoas com deficiência, crianças e idosos (Babá et al., 2015).

A zooterapia que é a utilização de animais para o tratamento de crianças e pessoas com alguma deficiência, e vem sendo muito utilizada para ajudar na superação de traumas físicos ou psicológicos gerando resultados satisfatórios, minimizando o sofrimento e proporcionando para a mente sensações de bondade (Porto \& Cassol, 2007). Com todos esses benefícios que o convívio com os animais vem trazendo a humanidade, os cães e os gatos estão sendo considerados parte da família (Lancendorfer et al., 2008).

A segurança dos animais de companhia vem se tornando muito discutido, já que esses animais fazem parte da família e do orçamento mensal, os tutores querem que seu pet tenha maior longevidade, que esteja saudável e que se sinta feliz. Nesse cenário o assunto mais questionável é a nutrição e a alimentação adequada, sendo um dos principais motivos pelo qual os tutores prefiram adquirir uma dieta 
natural para seus animais, afim de que eles tenham uma vida mais aproximada da sua natureza (Carciofi \& Jeremias, 2010; Groot \& Shreuder, 2009).

A alimentação natural vem conquistando o mundo dos animais pet principalmente por não conter produtos químicos, conservantes artificiais e a matéria prima ser de fácil aquisição, além disso a base da saúde é a nutrição, sendo a chave principal para manutenção da vida dos pequenos animais e essa escolha dos alimentos a serem oferecidos aos cães e gatos depende da preferência e da atitude de cada tutor que deve buscar pela ajuda de um profissional capacitado na área (Bragança \& Queiroz, 2020; Lopes et al., 2019).

Os produtos que compõem uma alimentação natural para cães e gatos no geral são os mesmos utilizados na alimentação dos seres humanos, como as carnes, ovos, frutas, verduras, miúdos, podem conter ossos ou não (se conter ossos eles devem estar crus) e se evita os alimentos altamente processados, essa deve-se ser formulada de acordo com as normas e exigências de animais de companhia (Buff et al., 2014). Mesmo com este contexto ainda existe tutores que preferem fazer as dietas em casa, e este tipo de alimentação é classificado como dieta caseira/home-prepared (Steiff \& Bauer, 2001). Os tutores que desejam obter uma dieta natural e balanceada, devem procurar o auxílio de um profissional, pois o preparo incorreto pode ocasionar obesidade, desnutrição ou agravar alguma doença. Desta forma, antes de preparar uma dieta em casa para os pets é muito importante que se consulte um nutricionista especializado em animais de companhia para realizar uma avaliação do animal.

\section{Material e métodos}

Essa pesquisa se caracterizou por uma abordagem qualitativo/quantitativo e é denominada como descritiva a partir do método de survey através da aplicação de um questionário virtual na plataforma Survio. O estudo foi realizado no estado de Rondônia, alcançando diversas cidades do estado, a coleta das respostas ocorreu nos meses de junho e julho de 2020. Os dados foram adquiridos por meio de plataforma virtual. Se constituiu a partir de questionário sobre o perfil dos tutores, a população de cães e gatos e a importância da consulta com um profissional na visão do tutor.

O público alvo desta pesquisa foi pessoas que fossem tutoras de pelo menos um animal sendo estes cães ou gatos, para obter uma amostra de população de pets por tutor. $\mathrm{O}$ trabalho dispôs de 3 questões sobre o perfil do tutor (sexo, faixa etária e renda por salário mínimo), duas questões para saber a população de cães/gatos e três últimas questões sobre a importância da nutrição na visão do tutor (se o tutor já consultou algum profissional em nutrição pet, se o mesmo gostaria de levar o seu animal ao nutricionista para obter uma dieta balanceada e quantos ele estaria disposto a pagar por uma consulta).

\section{Resultados e discussão}

Foram obtidas 109 respostas, sendo que a amostra populacional avaliada foi composta por $68,8 \%$ de mulheres ( 75 respostas), 28,4\% de homens ( 31 respostas) e 2,8\% (3 respostas) tutor não binário (Tabela 1). Em uma pesquisa realizada por Gouvêa (2019) verificou-se que tutores de cães e gatos a nível Brasil, onde de 434 respostas obtidas, 379 foram respondidas pelo sexo feminino (87\%), 54 pelo sexo masculino (12\%) e um tutor não binário $(0,2 \%)$. A partir desses dados observa-se que mulheres possuem mais animais domiciliados que homens.

A partir dos dados obtidos pode-se observar que a maior parte das pessoas que participaram da pesquisa são jovens com idade entre 18 e 33 anos. Esse resultado pode ter ocorrido pela facilidade dos jovens aos meios digitais (Tabela 1). Em uma pesquisa observou um baixo número de respondentes acima de 61 anos, o que possa ser explicado é que foi pela forma de divulgação da pesquisa pelas redes sociais e e-mail, cujos meios não são de fácil acesso para esta faixa etária (Gouvêa, 2019).

A maior parte do grupo amostral recebe entre 1 e 2 salários mínimos (60,6\%), e isso é uma realidade no estado de Rondônia, levando em consideração que as maiores fontes de empregos estão nos comércios, mercados e lojistas. De acordo com o aumento da renda salarial diminui a porcentagens de pessoas que os recebem, sendo que esses outros grupos se encaixam as pessoas concursadas, gerentes e proprietário dos comércios (Tabela 1). Mais o salário do indivíduo não influencia significativamente quando o assunto é saúde e bem-estar dos animais, sendo que a maior preocupação dos tutores é ter animais com maior longevidade e saúde. Um grupo entrevistado em uma pesquisa no Brasil teve sua 
maior parcela de renda salarial entre 2 e 3 salários mínimos somando um total de $37,1 \%$ da amostra total (Gouvêa, 2019). Na tabela 2 pode-se observar a proporção de cães em relação aos gatos.

Tabela 1. Perfil do tutor de animais de companhia no estado de Rondônia

\begin{tabular}{lcc}
\hline Sexo do tutor & Ocorrência & Proporção \% \\
\hline Feminino & 75 & 68,8 \\
Masculino & 31 & 28,4 \\
Não binário & 3 & 2,8 \\
\hline Faixa etária & Ocorrência & Proporção \% \\
\hline 18 a 25 anos & 50 & 20,9 \\
26 a 33 anos & 22 & 15,6 \\
34 a 41 anos & 17 & 11 \\
42 a 45 anos & 12 & 7,3 \\
=/> 50 anos & 8 & Proporção \% \\
\hline Renda por salário mínimo & Ocorrência & 60,6 \\
1 a 2 salários & 66 & 20,2 \\
3 a 4 salários & 22 & 12,8 \\
5 a 6 salários & 14 & 2,8 \\
7 a 8 salários & 3 & 3,7 \\
=/> 9 salários & 4 & \\
\hline
\end{tabular}

Tabela 2. Proporção de cães e gatos domiciliados para cada tutor estado de Rondônia

\begin{tabular}{lcc}
\hline Quantidade de cães & Ocorrência & Proporção \% \\
\hline 0 & 8 & 7,3 \\
1 a 2 & 68 & 62,4 \\
3 a 4 & 27 & 24,8 \\
5 a 6 & 5 & 4,6 \\
$=/>7$ & 1 & 0,9 \\
\hline Quantidade de gatos & Ocorrência & Proporção \% \\
\hline 0 & 63 & 57,8 \\
1 a 2 & 32 & 29,4 \\
3 a 4 & 6 & 5,5 \\
5 a 6 & 4 & 3,7 \\
$=/>7$ & 4 & 3,7 \\
\hline
\end{tabular}

Atualmente o mercado começa a abrir as portas para os felinos, com uma alimentação mais sofisticada, apesar desse contexto ainda é possível verificar que os gatos são os mais preteridos. Notase que das 109 pessoas entrevistadas apenas 8 indivíduos não tinham cães $(7,3 \%)$ e já para os gatos observa-se que 63 pessoas $(57,8 \%)$ não possuem essa espécie. Observa-se que por desconhecer o comportamento natural dos felinos haja recusa em tutoria destes. Sobre essa espécie que é considerada a mais limpa entre os animais de estimação, eles se lambem frequentemente, se lava após fazer suas necessidades e voltar do jardim, após brincar e também após ser tocado por uma pessoa (Ogoshi et al., 2015). As demais respostas foram 68 pessoas possuem de um a dois cães domiciliados, obteve 27 respostas para três a quatro animais, 5 pessoas responderam de cinco a seis e igual ou mais que sete cães foi 1 resposta. Já as demais respostas para a proporção de gatos domiciliados foram 32 pessoas possuem de um a dois animais, de três a quatro gatos foram 6 respostas, de cinco a seis foram 4, e igual ou maior que sete gatos obtiveram 4 respostas. Conforme Gouvêia, (2019) quando se trata de animais domiciliados, 39,6\% possuíam pelo menos um cão, já em relação aos gatos esta parcela foi menor $16 \%$. O IBGE (2020) corrobora esses dados, ao verificar que $44 \%$ de unidades domiciliares possuíam pelo menos um cachorro, e $17 \%$ de domicílios possuíam pelo menos um gato, sendo a região de maior proporção a região sul do país.

Nas redes sociais existem diversos profissionais zootecnistas que atuam na nutrição de cães e gatos. Apesar de formulação e balanceamento de dietas ser uma área do zootecnista, no estado de Rondônia, pode-se notar que existem mais veterinários que atuam nessa área em relação aos zootecnistas; porém, a maioria dos tutores nunca consultou um profissional em nutrição pet para obter informações sobre $o$ que melhor se adequa a dieta deles. A tabela 3 trata-se também sobre quanto o tutor estaria disposto a pagar para obter uma consulta e uma dieta balanceada para seu pet. A maioria dos tutores disseram 50,00 reais sendo um total de 38 respostas $(34,9 \%), 100,00$ reais obteve um total de 28 respostas $(25,7 \%)$, 150,00 reais um total de $13(11,9 \%), 200,00$ reais um total de $5(4,6 \%)$, igual ou maior que 250,00 reais 
obtiveram também cinco respostas $(4,6 \%)$ e 20 pessoas disseram que não pagaria nada (18,3\%). A Associação Brasileira da Indústria de Produtos para Animais de Estimação (ABINPET, 2018), revelou que os custos para manutenção cães de até $10 \mathrm{~kg}$ alcançam cerca de $\mathrm{R}$ \$216,20 mensais enquanto que para animais de grande porte esse valor chegaria a $\mathrm{R} \$ 411,32$ mensais.

Tabela 3. Frequência em que o tutor procurou por um profissional e qual a opinião do mesmo em relação a esse balanceamento nutricional para seu pet

\begin{tabular}{lcc}
\hline Você já consultou algum profissional em nutrição pet & & Proporção \% \\
\hline Alternativas & Ocorrência & 24,8 \\
\hline Sim, um veterinário & 27 & 4,6 \\
Sim, um zootecnista & 5 & 70,6 \\
Não & 77 & \\
\hline
\end{tabular}

Você gostaria de levar o seu animal ao nutricionista para obter uma dieta balanceada de acordo com a necessidade dele (idade, peso, nível de treinamento)

\begin{tabular}{lcc} 
Alternativas & Ocorrência & Proporção \% \\
\hline Sim & 75 & 68,8 \\
Não & 34 & 31,2 \\
\hline Quantos você pagaria por uma consulta assim & & Proporção \% \\
\hline Alternativas & Ocorrência & 34,9 \\
R\$ 50,00 & 38 & 25,7 \\
R 100,00 & 28 & 11,9 \\
R 150,00 & 13 & 4,6 \\
R 200,00 & 5 & 4,6 \\
R $=/>250,00$ & 5 & 18,3 \\
Nada & 20 & \\
\hline
\end{tabular}

\section{Conclusão}

Foi observado a importância do perfil do tutor em relação a população de cães e gatos, ainda a conscientização sobre a necessidade de consultar um profissional antes de adquirir qualquer dieta nova no mercado para manter a saúde e o bem-estar dos animais de companhia, e como temos as dietas alternativas que vem se tornando tendência é importante conhecer o nível de aceitabilidade do tutor para esse tipo de alimentação.

\section{Referências bibliográficas}

ABINPET. (2021). Caderno especial Abinpet-Associação Brasileira da Indústria de Produtos para Animais de Estimação. Agro Analysis, 35(1), 35-40.

Babá, A. Y., Obara, A. T., \& Silva, E. S. (2015). Levantamento do conhecimento de proprietários de cães domésticos sobre zoonoses. UNOPAR - Científica Ciências Humanas e Educação, 14(3), 251258.

Bragança, D. R., \& Queiroz, E. O. (2020). Manejo nutricional de cães e gatos e a inserção de alimentos alternativos na dieta de pequenos animais domiciliados no estado de Rondônia, Brasil. Brazilian Journal of Development, 6(10), 75090-75098. https://doi.org/10.34117/bjdv6n10-074.

Buff, P. R., Carter, R. A., Bauer, J. E., \& Kersey, J. H. (2014). Natural pet food: A review of natural diets and their impact on canine and feline physiology. Journal of Animal Science, 92(9), 3781-3791. https://doi.org/10.2527/jas.2014-7789.

Carciofi, A. C., \& Jeremias, J. T. (2010). Progresso científico sobre nutrição de animais de companhia na primeira década do século XXI. Revista Brasileira de Zootecnia, 39, 35-41.

Gouvêa, F. L. (2019). Alimentos convencionais e a tendência a alimentos alternativos para animais de companhia: uma visão sobre o perfil de tutores e a escolha de alimentos para cães e gatos.

Groot, J., \& Shreuder, W. (2009). Biological, naturally logical. Amsterdam: AFB International.

IBGE. Pesquisa Nacional de Saúde. 2020. Disponível em: http://www.ibge.gov.br/home/estatistica/popul acao/pns/2013/. Acesso 20 de abr. 2020.

Lancendorfer, K. M., Atkin, J. L., \& Reece, B. B. (2008). Animals in advertising: Love dogs? Love the ad! Journal of Business Research, 61(5), 384-391.

Lima, A. F. M., \& Luna, S. P. L. (2012). Algumas causas e consequências da superpopulação canina e 
felina: acaso ou descaso? Revista de Educação Continuada Em Medicina Veterinária e Zootecnia, $10(1), 32-38$.

Lopes, L. A., Lira, R. C., Camargo, K. S., \& Santos, E. L. (2019). Manejo nutricional de cães e gatos domiciliados no município de Maceió, Alagoas, Brasil. Revista de Educação Continuada Em Medicina Veterinária e Zootecnia Do CRMV-SP, 17(3), 36-40.

Ogoshi, R. C. S., Reis, J. S., Zangeronimo, M. G., \& Saad, F. M. O. B. (2015). Conceitos básicos sobre nutrição e alimentação de cães e gatos. Ciência Animal, 25(1), 64-75.

Porto, R. T. C., \& Cassol, S. (2007). Zooterapia uma lição de cidadania: o cão sociabilizador e a criança vítima de violência intrafamiliar. Discurso Jurídico, 3(2), 46-74.

Steiff, E. L., \& Bauer, J. E. (2001). Nutritional adequacy of diets formulated for companion animals. Journal of the American Veterinary Medical Association, 219(5), 601-604. https://doi.org/10.2460/javma.2001.219.601.

Histórico do artigo:

Recebido: 12 de abril de 2021

Aprovado: 28 de maio de 2021
Licenciamento: Este artigo é publicado na modalidade Acesso Aberto sob a licença Creative Commons Atribuição 4.0 (CC-BY 4.0), a qual permite uso irrestrito, distribuição, reprodução em qualquer meio, desde que o autor e a fonte sejam devidamente creditados. 